\title{
MULTIRATE DIGITAL DECENTRALIZED CONTROL DESIGN
}

\author{
Seung-Hi Lee* \\ * Samsung Advanced Institute of Technology, Suwon, KOREA \\ shl@sait.samsung.co.kr
}

\begin{abstract}
Multirate digital decentralized control design is presented for linear timeinvariant systems with interaction between subsystems. The design technique, implemented in multirate digital control, eventually allows us to separately design controllers for each subsystem without considering interaction between them. The resulting closed-loop system with interaction between subsystems being eliminated delivers the same desired closed-loop responses each subsystem produces, that can be expected in the design stage. By this we mean that the resulting controller eliminating the interaction between the subsystems delivers desired closed-loop responses in the existence of interaction between subsystems. Copyright(C)2005 IFAC
\end{abstract}

Keywords: Multirate control, Digital control, Decentralized control.

\section{INTRODUCTION}

Interaction between subsystems is very problematic in many control designs to obtain desired closed-loop responses, that causes most likely very different results from what we expect. Such problem becomes even more difficult for very large scale systems. Although stabilization of such coupled systems seems to be not difficult (see (Araki, 1993) and references therein), controller design to attain desired responses becomes more challenging. Conventionally, separate control loops are designed for each subsystem. However, each subsystem interacts internally and thereby the controller (designed neglecting such interaction) yields very different (more likely unpredictable) responses. If the dynamic coupling is not negligible, the resulting closed-loop system will exhibit performance degradation and even become unstable. Thereby control systems need to be designed considering the dynamic interaction between subsystems. In general, however, such design technique is very complex and actual implementation also becomes very difficult.
Control design for a plant with interaction between subsystems to attain desired subsystems' responses is very challenging over the decentralized stabilization problem. The single stage direct closed-loop dynamics assignment strategy mentioned above is not easy to apply. The fundamental problem of decentralized control has been to provide conditions under which a system can be stabilized using local controllers. The stabilization problem of a plant with independent decentralized controllers has been widely studied (e.g. (Wang and Davison, 1973; Corfmat and Morse, 1976; Sezer and Šiljak, 1981; Özgüler, 1990; Khargonekar and Özgüler, 1994; Sebe, 2003)). Digital solutions such as multirate decentralized controllers have been investigated in (Sezer and Šiljak, 1990; Ito, 1997; Longhi, 1994; Ciferri and Longhi, 2003). So far, however, there has been no work considering a decentralized control design for desired responses over the stabilization problem.

This paper presents a multirate digital decentralized control design for linear time-invariant systems with interaction between subsystems. The 
problem considered in the paper is primarily the elimination of interaction between the subsystems and the design of a controller such that the desired performance is achieved. The design technique, implemented in multirate digital control, must allow us to separately design controllers for each subsystem without considering the dynamic interaction between them. The resulting closedloop system with interaction between subsystems being eliminated must deliver the same desired closed-loop responses each subsystem produces, that can be expected in the design stage. By this we mean that the decentralized feedback control, eliminating the interaction between the subsystems, must deliver desired closed-loop responses in the existence of interaction between subsystem.

The proposed design technique uses one of many potential capabilities of multirate control: it can deliver an expanded spanning of the system's input matrix (or observation matrix dependent on where the multirate sampling is applied). By using multirate control, a full row rank input matrix can be obtained, so that by a suitable (and easy to compute) feedback law, all the closed-loop dynamics can be freely assigned (see (Araki, 1993) and references therein). Then, a decentralizing feedback in order to simplify the stabilization of the plant can be obtained. Furthermore, the single stage direct closed-loop dynamics assignment strategy is available in the literature (e.g. (Kabamba and Yang, 1991)) over the two stage decoupling and stabilization strategy.

Throughout the paper, the notation $M^{+}$denotes the pseudo inverse matrix of $M$ such that $M M^{+} M=M$. Also, although not mentioned individually, all the matrix dimensions are assumed to be appropriate for compatible matrix formulations.

\section{PROBLEM FORMULATION}

To begin with, consider a real linear timeinvariant system model with interaction between $n_{s}$ subsystems described by

$$
\dot{x}=A x+B u
$$

where

$$
\begin{aligned}
& A \in \mathbb{R}^{n \times n}=\left[A_{(l m)}\right]_{l, m=1, \cdots, n_{s}}, \\
& B \in \mathbb{R}^{n \times n_{u}}=\left[B_{(l m)}\right]_{l, m=1, \cdots, n_{s}} .
\end{aligned}
$$

The matrix elements $A_{(l m)}$ and $B_{(l m)}$ describe interaction between subsystems $\left(A_{(l l)}, B_{(l l)}\right)$ and $\left(A_{(m m)}, B_{(m m)}\right)$ for $l \neq m, l, m=1, \cdots, n_{s}$. The subsystems $\left(A_{(l l)}, B_{(l l)}\right), l=1, \cdots, n_{s}$, each have their dimensions, while the matrix elements $A_{(l m)}$ and $B_{(l m)}, l, m=1, \cdots, n_{s}$, must have compatible dimensions. Throughout the paper, it is assumed that all the subsystem doublets
$\left(A_{(l l)}, B_{(l l)}\right), l=1, \cdots, n_{s}$, are controllable (and thereby so is the system doublet $(A, B)$ ), and all the state measurements are available for feedback.

Interaction between subsystems is very problematic in many control designs to obtain desired closed-loop responses. Many times, however, we need to design a control system for such plants. Conventional decentralized control design was focused on closed-loop stability. Although stabilization of such plant seems to be not difficult (as shown in the references (Wang and Davison, 1973; Corfmat and Morse, 1976; Sezer and Šiljak, 1981; Özgüler, 1990; Khargonekar and Özgüler, 1994; Sebe, 2003; Sezer and Šiljak, 1990; Ito, 1997; Longhi, 1994; Ciferri and Longhi, 2003), controller design to attain desired closed-loop responses becomes very challenging. In the presence of dynamic interaction between subsystems, the resulting stable closed-loop system most likely exhibits serious performance degradation and even unstable responses.

For a nonpathological sampling period $T_{s}$, the zero-order-holder $(\mathrm{ZOH})$ discrete-time equivalent (Franklin et al., 1990) becomes

$$
x(k+1)=\Phi x(k)+\Gamma u
$$

where $\Phi=e^{A T_{s}}, \Gamma=\int_{0}^{T_{s}} e^{A\left(T_{s}-\eta\right)} B d \eta$. Also, the subsystems $\left(A_{(l l)}, B_{(l l)}\right), l=1, \cdots, n_{s}$, each have their discrete-time equivalents

$$
\begin{aligned}
& \Phi_{(l l)}=e^{A_{(l l)} T_{s}}, \\
& \Gamma_{(l l)}=\int_{0}^{T_{s}} e^{A_{(l l)}\left(T_{s}-\eta\right)} B_{(l l)} d \eta
\end{aligned}
$$

for $l=1, \cdots, n_{s}$ such that

$$
\begin{aligned}
& \hat{\Phi}=\operatorname{diag}\left(\Phi_{(11)}, \cdots, \Phi_{\left(n_{s} n_{s}\right)}\right), \\
& \hat{\Gamma}=\operatorname{diag}\left(\Gamma_{(11)}, \cdots, \Gamma_{\left(n_{s} n_{s}\right)}\right) .
\end{aligned}
$$

Then, the differences expressed by $\Delta \Phi=\Phi-\hat{\Phi}$ and $\Delta \Gamma=\Gamma-\hat{\Gamma}$ represent interaction between the subsystems. Applying the control gain $K=$ $\operatorname{diag}\left(K_{(1)}, \cdots, K_{\left(n_{s}\right)}\right)$ determined for the system $(\hat{\Phi}, \hat{\Gamma})$ to the system $(\Phi, \Gamma)$ thereby yields the closed-loop $\Phi-\Gamma K=\hat{\Phi}-\hat{\Gamma} K+\Delta \Phi-\Delta \Gamma K$ most likely producing very different results. Observe $\Delta \Phi-\Delta \Gamma K$ makes the closed-loop response fairly different from what we expect.

The problem considered in the paper is primarily the elimination of interaction between the subsystems and the design of a controller such that the desired performance is achieved. By this we mean that the resulting controller eliminating the interaction between the subsystems must deliver desired closed-loop responses in the existence of interaction between subsystems, that can be expected in the design stage from each subsystem response. 


\section{MULTIRATE DECENTRALIZED CONTROL DESIGN}

\subsection{Multirate digital control scheme}

Consider a multirate discrete-time equivalent of (1) expressed by

$$
x(k, i+1)=\Phi_{f} x(k, i)+\Gamma_{f} u(k, i)
$$

where $\Phi_{f}=e^{A T_{c}}, \Gamma_{f}=\int_{0}^{T_{c}} e^{A\left(T_{c}-\eta\right)} B d \eta$ and $T_{c}$ is the nonpathological control update period and $R_{u}$ is the input multiplicity such that the sampling update period $T_{s}=T_{c} R_{u}$. The indexes $k$ and $i$ indicate measurement update and control update instants, respectively, such that $t=(k+$ $\left.i / R_{u}\right) T_{s}$ for $i=0,1, \cdots, R_{u}-1$. The lifting technique (Kranc, 1957; Meyer, 1992; Albertos and Salt, 1999) can be applied to represent the multirate system as a time-invariant lifted finitedimensional discrete system. Using the lifting technique, we can represent the plant as a lifted time-invariant system in control update period $T_{c}$ as

$$
x(k+1,0)=\tilde{\Phi} x(k, 0)+\tilde{\Gamma} \tilde{u}(k, 0)
$$

where

$$
\tilde{\Phi}=\Phi_{f}^{R_{u}}, \quad \tilde{\Gamma}=\left[\begin{array}{lll}
\Phi_{f}^{R_{u}-1} \Gamma_{f} & \cdots & \Gamma_{f}
\end{array}\right] .
$$

Corollary 1. The doublet $(\tilde{\Phi}, \tilde{\Gamma})$ is controllable for any $R_{u} \geq 1$ if $\left(\Phi_{f}, \Gamma_{f}\right)$ is controllable.

Proof: Build the controllability matrix of $(\tilde{\Phi}, \tilde{\Gamma})$ and use the controllability matrix of $\left(\Phi_{f}, \Gamma_{f}\right)$. Then, it becomes apparent.

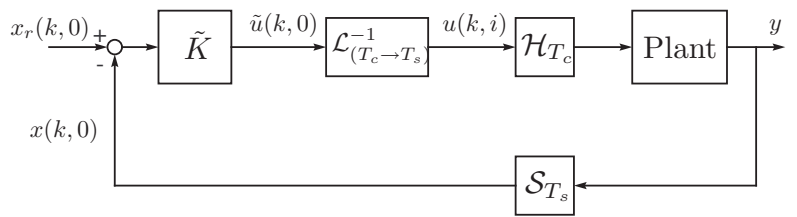

Fig. 1. Multirate state feedback control system

Using the lifting operator

$$
\mathcal{L}_{\left(T_{c} \rightarrow T_{s}\right)}: l_{T_{c}}^{p} \rightarrow l_{T_{s}}^{p}, \quad 1 \leq p \leq \infty
$$

we build a multirate digital control scheme (Fig. 1) (Lee, submitted)

$$
u(k, i)=\mathcal{L}_{\left(T_{c} \rightarrow T_{s}\right)}^{-1} \tilde{u}(k, 0)
$$

where

$$
\begin{aligned}
\tilde{u}(k, 0) & =\left[\begin{array}{c}
u(k, 0) \\
u(k, 1) \\
\vdots \\
u\left(k, R_{u}-1\right)
\end{array}\right] \\
& =\tilde{K}\left(x_{r}(k, 0)-x(k, 0)\right) \\
& =\tilde{K}\left(N_{x} r(k, 0)-x(k, 0)\right)
\end{aligned}
$$

where $\tilde{K}$ is the multirate control gain to be determined for improved inter-sample behavior.

\subsection{Multirate decentralizing state feedback control}

To begin with, we let

$$
\begin{aligned}
& \hat{\Phi}_{f}=\operatorname{diag}\left(\Phi_{f,(11)}, \cdots, \Phi_{f,\left(n_{s} n_{s}\right)}\right), \\
& \hat{\Gamma}_{f}=\operatorname{diag}\left(\Gamma_{f,(11)}, \cdots, \Gamma_{f,\left(n_{s} n_{s}\right)}\right)
\end{aligned}
$$

and their lifted expressions

$$
\tilde{\hat{\Phi}}=\hat{\Phi}_{f}^{R_{u}}, \quad \tilde{\hat{\Gamma}}=\left[\hat{\Phi}_{f}^{R_{u}-1} \hat{\Gamma}_{f} \cdots \hat{\Gamma}_{f}\right]
$$

where

$$
\begin{aligned}
\Phi_{f,(m m)} & =e^{A_{(m m)} T_{c}}, \\
\Gamma_{f,(m m)} & =\int_{0}^{T_{c}} e^{A_{(m m)}\left(T_{c}-\eta\right)} B_{(m m)} d \eta
\end{aligned}
$$

for $m=1, \cdots, n_{s}$.

Assumption 1. The state feedback control gain matrix $\hat{K}=\operatorname{diag}\left(K_{(1)}, \cdots, K_{\left(n_{s}\right)}\right)$ is determined for the system $(\hat{\Phi}, \hat{\Gamma})$ such that each subsystem closed-loop described by $\Phi_{f,(m m)}-\Gamma_{f,(m m)} \hat{K}_{(m)}$, $m=1, \cdots, n_{s}$, produces desired responses.

Assumption 2. For a control input dimension $n_{u}$ and a state dimension $n$, the input multiplicity $R_{u}$ satisfies the condition $n_{u} R_{u} \geq n$.

The multirate state feedback gain $\tilde{\hat{K}}$ needs to be determined for improved inter-sample behavior (e.g. no ripples).

Proposition 1. Given the control gain $\hat{K}$ designed for $\left(\hat{\Phi}_{f}, \hat{\Gamma}_{f}\right)$, the multirate state feedback control gain $\tilde{\hat{K}}$ for $(\tilde{\hat{\Phi}}, \tilde{\hat{\Gamma}})$ computed from

$$
\tilde{\hat{K}}=(\tilde{\hat{\Gamma}})^{+}\left(\tilde{\hat{\Phi}}-\left(\hat{\Phi}_{f}-\hat{\Gamma}_{f} \hat{K}\right)^{R_{u}}\right)
$$

delivers discrete closed-loop matching with $\hat{\Phi}_{f}-$ $\hat{\Gamma}_{f} \hat{K}$ at every control instant.

Proof: Appears in (Lee, submitted).

Directly determining the control gain $\tilde{\hat{K}}$ for $\left(\tilde{\hat{\Phi}}_{f}, \tilde{\hat{\Gamma}}_{f}\right)$ is not reliable in the case of complex conjugate eigenvalue assignment. The gain and the resulting closed-loop can become complex valued. Also, even real valued control gain can cause $(\tilde{\hat{\Phi}}-\tilde{\hat{\Gamma}} \hat{\hat{K}})^{1 / R_{u}}$ to be complex valued for $R_{u}>1$, while the closed-loop $\hat{\Phi}_{f}-\hat{\Gamma}_{f} \hat{K}$ must be real producing (complex conjugate) eigenvalues. Accordingly, the stated "free closed-loop dynamics assignment" by a suitable feedback law" 
(Araki, 1993) is in general not possible. The multirate control gain $\tilde{\hat{K}}$ thereby in general must be computed indirectly as in (5).

The multirate state feedback control gain produces discrete closed-loop state matching and thereby predictable response at each control update instant. Increasing the control update rate thereby tends to produce improved inter-sample behavior of the closed-loop system (Lee, submitted).

Applying the state feedback control law $-\tilde{\hat{K}} x(k)$ with $\tilde{\hat{K}}$ computed from $(5)$ to $(\tilde{\Phi}, \tilde{\Gamma})$ delivers a closed-loop expressed by $\tilde{\Phi}-\tilde{\Gamma} \tilde{\hat{K}}$ that most likely yields very different and even becomes unstable behavior. A candidate to resolve such design complexity in the design of controller for a plant with interaction between subsystems is to use feedback control eliminating the interaction between subsystems such that the resulting closed-loop exhibits $\tilde{\Phi}-\tilde{\Gamma} \bar{K}=\tilde{\hat{\Phi}}-\tilde{\hat{\Gamma}} \tilde{\hat{K}}$. Then, the resulting closed-loop system must preserve closed-loop stability and deliver the same desired closed-loop response (with an appropriate reference mapping) each subsystem produces in combination. Then, the design technique provides not only stability but also desired responses in the existence of interaction between subsystems.

Proposition 2. Suppose that a discrete-time plant model (3), with interaction between subsystems, is given and the state feedback control gain $\hat{K}$ is determined such that each subsystem closed-loop produces desired responses. Then, the multirate digital decentralized state feedback control law

$$
\begin{aligned}
u(k, i) & =\mathcal{L}_{\left(T_{c} \rightarrow T_{s}\right)}^{-1} \tilde{u}(k, 0) \\
& =\mathcal{L}_{\left(T_{c} \rightarrow T_{s}\right)}^{-1} \bar{K}\left(\bar{N}_{x} r(k, 0)-x(k, 0)\right)
\end{aligned}
$$

with

$$
\bar{K}=(\tilde{\Gamma})^{+}(\tilde{\Phi}-(\tilde{\hat{\Phi}}-\tilde{\hat{\Gamma}} \tilde{\hat{K}}))
$$

and

$$
\bar{N}_{x}=(\tilde{\Gamma} \bar{K})^{+} \tilde{\hat{\Gamma}} \tilde{\hat{K}} N_{x}
$$

delivers a decentralized closed-loop system matrix

$$
\tilde{\Phi}-\tilde{\Gamma} \bar{K}=\tilde{\hat{\Phi}}-\tilde{\hat{\Gamma}} \tilde{\hat{K}}
$$

preserving closed-loop stability. Furthermore, the resulting closed-loop system $\left(\tilde{\Phi}-\tilde{\Gamma} \bar{K}, \tilde{\Gamma} \bar{K} \bar{N}_{x}\right)$ must yields the same desired closed-loop response $\left(\tilde{\hat{\Phi}}-\tilde{\hat{\Gamma}} \tilde{\hat{K}}, \tilde{\hat{\Gamma}} \tilde{\hat{K}} N_{x}\right)$ produces, which in fact consists of the desired responses of each subsystem.

Proof: Simply introducing the state feedback control $-\bar{K} x(k, 0)$ with $\bar{K}$ in $(7)$ exhibits

$$
\begin{aligned}
\tilde{\Phi}-\tilde{\Gamma} \bar{K} & =\tilde{\Phi}-\tilde{\Gamma}(\tilde{\Gamma})^{+}(\tilde{\Phi}-(\tilde{\hat{\Phi}}-\tilde{\hat{\Gamma}} \tilde{\hat{K}})) \\
& =\tilde{\hat{\Phi}}-\tilde{\hat{\Gamma}} \tilde{\hat{K}} .
\end{aligned}
$$

Also, simply applying $\tilde{u}(k, 0)=\bar{K}\left(\bar{N}_{x} r(k, 0)-x(k, 0)\right)$ with $\bar{K}$ and $\bar{N}_{x}$ given in (7) and (8), respectively, yields

$$
\begin{aligned}
x(k+1,0) & =\tilde{\Phi} x(k, 0)+\tilde{\Gamma} \tilde{u}(k, 0) \\
& =\tilde{\Phi} x(k, 0)+\tilde{\Gamma} \bar{K}\left(\bar{N}_{x} r(k, 0)-x(k, 0)\right) \\
& =(\tilde{\hat{\Phi}}-\tilde{\hat{\Gamma}} \tilde{\hat{K}}) x(k, 0)+\tilde{\hat{\Gamma}} \tilde{\hat{K}} N_{x} r(k, 0) .
\end{aligned}
$$

The resulting closed-loop, with interaction between the subsystems being eliminated, must deliver desired closed-loop response.

The resulting controller, eliminating the interaction between the subsystems, must deliver desired closed-loop responses (not only stability but also desired responses) in the existence of interaction between subsystem. Also, such responses in fact can be expected from the desired responses of each subsystem. Applying the proposed control design technique, separate controller design is thereby possible for each subsystem without considering the dynamic interaction between them.

The condition $n_{u} R_{u} \geq n$ given in Assumption 2 delivers a state feedback gain $\bar{K}$ in (7) which exhibits exact decentralization as shown in (9). Otherwise, the solution in the least squares sense is obtained, which does not exhibit the exactly decentralized closed-loop system. Also, due to the numerical reliability problems, decentralized control design for ill-conditioned plants with interaction between subsystems possibly results in an imperfect decentralization.

The final formulation in (7) allows an efficient simultaneous control design for stability and desired time responses: Given a plant with internally coupled subsystems, we find controller which achieves not only stability but also desired time response in the each subsystem. By this we mean that the controller delivers desired closed-loop response while compensating the interaction between the subsystems, that can be expected from each subsystem response. The proposed multirate digital state feedback decentralized control scheme is also applicable to multi-input multi-output systems. Furthermore, no ripple will exist at continuous process output because actually each fast rate control is being computed with links among them (Lee, submitted).

Actual control design using the proposed method is very simple as explained in the following design procedure:

Step 1) Build $\left(\Phi_{f}, \Gamma_{f}\right)$ and $\left(\hat{\Phi}_{f}, \hat{\Gamma}_{f}\right)$ and their multirate discrete-time equivalents $(\tilde{\Phi}, \tilde{\Gamma})$ and $\left(\tilde{\hat{\Phi}}_{f}, \tilde{\hat{\Gamma}}_{f}\right)$;

Step 2) Determine $\hat{K}$ for $\left(\hat{\Phi}_{f}, \hat{\Gamma}_{f}\right)$;

Step 3) Compute $\tilde{\hat{K}}$ from (5);

Step 4) Compute $\bar{K}$ from (7);

Step 5) Implement the multirate control in (6). 


\section{AN APPLICATION EXAMPLE}

Consider a simple continuous-time plant model with strong interaction between two subsystems:

$$
\dot{x}=A x+B u
$$

where

$$
\begin{aligned}
A & =\left[\begin{array}{ll}
A_{(11)} & A_{(12)} \\
A_{(21)} & A_{(22)}
\end{array}\right] \\
& =\left[\begin{array}{cccc}
0 & 1.0 & 0 & -0.1 \\
0 & 0 & 0 & 0 \\
0 & -0.5 & 0 & 1.0 \\
0 & 0 & -1.5 \times 10^{9} & -2.0 \times 10^{4}
\end{array}\right], \\
B= & {\left[\begin{array}{ll}
B_{(11)} & B_{(12)} \\
B_{(21)} & B_{(22)}
\end{array}\right]=\left[\begin{array}{cc}
0 & 0 \\
50000 & 50 \\
0 & 0 \\
-25000 & 1500
\end{array}\right] . }
\end{aligned}
$$

The system has interaction between the subsystems $\left(A_{(11)}, B_{(11)}\right)$ and $\left(A_{(22)}, B_{(22)}\right)$ represented by $A_{(21)}, B_{(12)}$, and $B_{(21)}$. Observe both subsystems affecting each other.

Let us consider a sampling period $T_{s}=100 \mu \mathrm{sec}$, and an input multiplicity $R_{u}=T_{s} / T_{c}=4$ such that the corresponding control period becomes $T_{c}=25 \mu \mathrm{sec}$. Then, we obtain the multirate discrete-time equivalent models expressed by $\left(\Phi_{f,(11)}, \Gamma_{f,(11)}\right),\left(\Phi_{f,(22)}, \Gamma_{f,(22)}\right)$, and $\left(\Phi_{f}, \Gamma_{f}\right)$.

Firstly, the control gains $K_{(m)}$ are determined for $\left(\Phi_{f,(m m)}, \Gamma_{f,(m m)}\right), m=1,2$, such that

$$
\begin{aligned}
\hat{K} & =\operatorname{diag}\left(K_{(1)}, K_{(2)}\right) \\
& =\left[\begin{array}{cccc}
417.3 & 0.1364 & 0 & 0 \\
0 & 0 & -9.547 \times 10^{5} & -15.908
\end{array}\right] .
\end{aligned}
$$

Then, its multirate version $\tilde{\hat{K}}$ is computed from (5) as

$$
\tilde{\hat{K}}=\left[\begin{array}{cccc}
413.56 & 0.13608 & 0 & 0 \\
0 & 0 & -1.1329 \times 10^{5} & 6.2618 \\
347.20 & 0.12303 & 0 & 0 \\
0 & 0 & -8.6356 \times 10^{5} & -28.138 \\
280.84 & 0.10998 & 0 & 0 \\
0 & 0 & -1.1294 \times 10^{6} & -53.209 \\
214.49 & 0.09693 & 0 & 0 \\
0 & 0 & -2.9751 \times 10^{5} & -34.704
\end{array}\right] .
$$

Finally, we compute the decentralized feedback control gain $\bar{K}$ from (7) as

$$
\bar{K}=\left[\begin{array}{cccc}
2015.9 & -0.86237 & 2.5296 \times 10^{3} & 1.7994 \\
305.11 & -0.40017 & 1.5946 \times 10^{4} & 1.4581 \\
-2213.6 & 2.5670 & -6.6837 \times 10^{4} & -7.5413 \\
185.58 & -0.17505 & 4.7079 \times 10^{3} & 0.5337 \\
743.84 & -1.8973 & 1.2668 \times 10^{5} & 9.5018 \\
-212.76 & 0.38574 & -1.8883 \times 10^{4} & -1.5666 \\
710.23 & 0.65804 & -6.2335 \times 10^{4} & -3.7570 \\
-634.31 & 0.88431 & -3.6907 \times 10^{4} & -3.2983
\end{array}\right]
$$

Then we must have $\tilde{\Phi}-\tilde{\Gamma} \bar{K}=\tilde{\hat{\Phi}}-\tilde{\hat{\Gamma}} \tilde{\hat{K}}$ with closedloop eigenvalues $0.66430 \pm 0.21716 i, 0.47435 \pm$ 0.16445i. On the other hand, the closed-loop $\tilde{\Phi}-\tilde{\Gamma} \tilde{\hat{K}}$ yields eigenvalues $0.88636 \pm 0.24942 i$, $0.25631 \pm 0.27959$. Although still in stable, the latter system is expected to produce fairly different responses.

To obtain desired plant output, we use

$$
N_{x}=\left[\begin{array}{llll}
1 & 0 & -1 & 0
\end{array}\right]^{T} .
$$

Then $\bar{N}_{x}$ can be computed from (8) as

$\bar{N}_{x}=\left[\begin{array}{llll}5.560 & -1.229 \times 10^{4}-0.5255 & -8.167 \times 10^{3}\end{array}\right]^{T}$.

Fig. 2 compares normalized step responses. Observe the contribution of the proposed decentralized feedback control. The decentralized feedback control contributes to produce the same time response $\left(\tilde{\hat{\Phi}}-\tilde{\hat{\Gamma}} \tilde{\hat{K}}, \tilde{\hat{\Gamma}} \tilde{\hat{K}} N_{x}\right)$ produces with the control gain $\tilde{\hat{K}}$ computed from $\hat{K}$ that is designed for $\left(\Phi_{f}, \Gamma_{f}\right)$ to obtain a desired closed-loop (Figs. 2(a) and $2(\mathrm{c}))$. On the other hand, simply applying the gain $\tilde{\hat{K}}$ to $(\tilde{\Phi}, \tilde{\Gamma})$, the system with interaction between subsystems, produces quite a different response (Fig. 2(b)).

\section{CONCLUSIONS}

This paper has presented multirate digital decentralized controller design for linear time-invariant systems with interaction between subsystems. The problem considered in the paper was primarily the elimination of interaction between the subsystems and the design of a controller such that the desired performance is achieved. Applying the proposed control design technique, implemented in multirate digital, separate controller design was possible for each subsystem without considering interaction between them. The resulting closed-loop system with interaction between subsystems being eliminated delivered the same desired closed-loop responses each subsystem produces, that can be expected in the design stage. By this we mean that the resulting controller eliminating the interaction between the subsystems must deliver desired closed-loop responses in the existence of interaction between subsystems. Application results demonstrated the utility of the proposed multirate digital decentralized feedback control. The decentralized feedback control, eliminating the interaction between the subsystems, delivered desired closed-loop responses in the existence of interaction between subsystem.

\section{REFERENCES}

Albertos, P. and J. Salt (1999). Receding horizon control of non-uniformly sampled data systems. In: Proc. American Control Conference. pp. 4300-4304. 


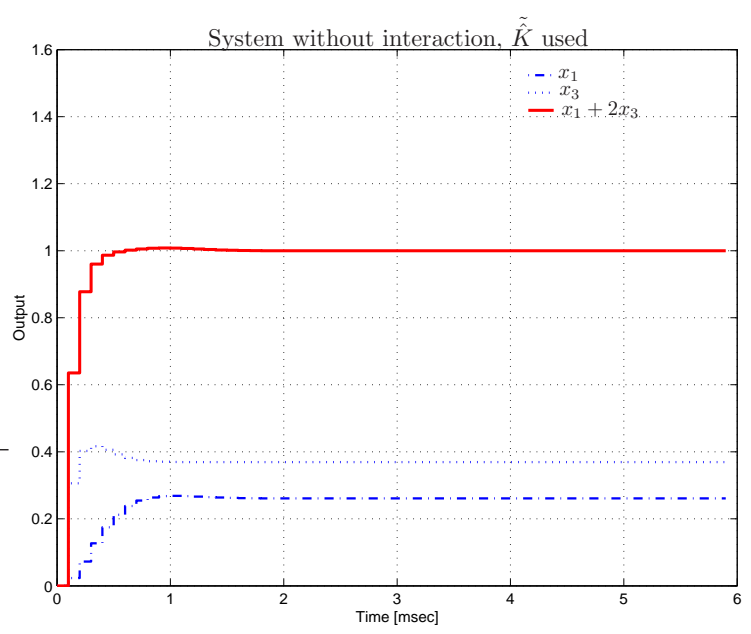

(a) $\tilde{\hat{K}}$ used for $(\tilde{\hat{\Phi}}, \tilde{\hat{\Gamma}})$

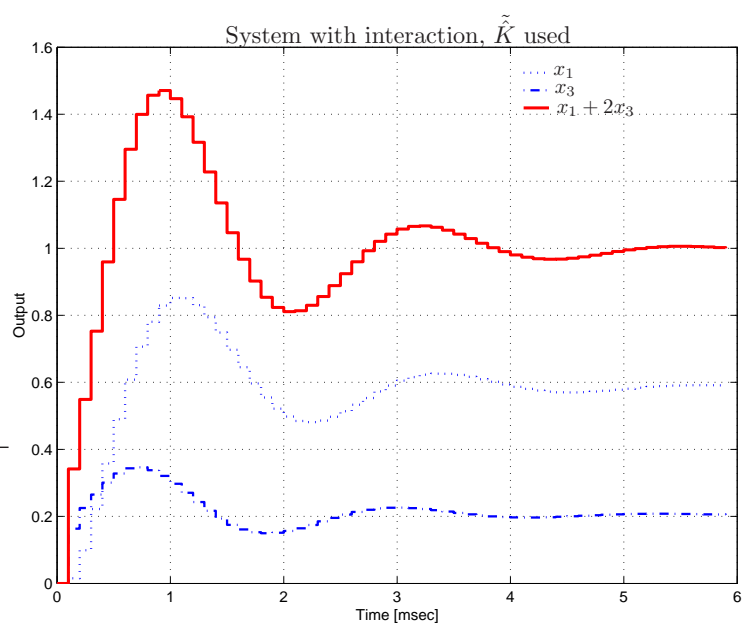

(b) $\tilde{\hat{K}}$ used for $(\tilde{\Phi}, \tilde{\Gamma})$

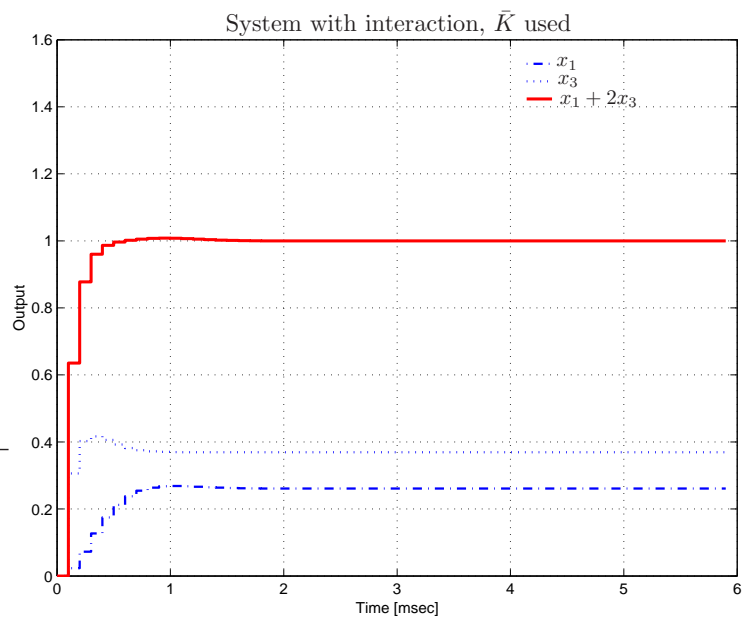

(c) $\bar{K}$ used for for $(\tilde{\Phi}, \tilde{\Gamma})$
Araki, M. (1993). Recent developments in digital control theory. In: IFAC World Congress. Vol. 9. pp. 251-260.

Ciferri, R. and S. Longhi (2003). Decentralized control of multirate sampled-data systems. In: Proc. IEEE Conference on Decision and Control. pp. 584-585.

Corfmat, J. P. and A. S. Morse (1976). Decentralized control of linear multivariable system. Automatica 12, 479-495.

Franklin, G. F., J. D. Powell and M. L. Workman (1990). Digital Control of Dynamic Systems. Addison Wesley.

Ito, H. (1997). Worst-case performance and stability of multirate sampled-data systems with nonsynchronous decentralized controllers. In: Proc. American Control Conference. pp. 778783.

Kabamba, P. T. and C. Yang (1991). Simultaneous controller design for linear timeinvariant systems. IEEE Trans. Automatic Control 36(1), 106-111.

Khargonekar, P. P. and A. B. Özgüler (1994). Decentralized control and periodic feedback. IEEE Trans. Automatic Control 39, 877-882.

Kranc, G. M. (1957). Input-output analysis of multirate feedback systems. IRE Trans. Automatic Control pp. 21-28.

Lee, S.-H. (submitted). Multirate digital control system design and its application to computer disk drives. IEEE Trans. Control Systems Technology.

Longhi, S. (1994). Structual properties of multirate sampled-data systems. IEEE Trans. Automatic Control 39, 692-696.

Meyer, D. G. (1992). Cost translation and a lifting approach to the multirate LQG problem. IEEE Trans. Automatic Control 37, 14111415.

Özgüler, A. B. (1990). Decentralized control: a stable proper fractional approach. IEEE Trans. Automatic Control 35(10), 1109-1117.

Sebe, N. (2003). A unified approach to decentralized controller design. In: Proc. IEEE Conference on Decision and Control. pp. 1086-1091.

Sezer, M. E. and D. D. Šiljak (1981). Structually fixed modes. Systems \& Control Letters 1, 6064.

Sezer, M. E. and D. D. Šiljak (1990). Decentralized nultirate control. IEEE Trans. Automatic Control 35, 60-65.

Wang, S. H. and E. J. Davison (1973). On the stabilization of decentralized control systems. IEEE Trans. Automatic Control AC18, 473-478.

Fig. 2. Step responses 